\title{
An Approach to Schema Integration Based on Transformations and Behaviour*
}

\author{
Christiaan Thieme and Arno Siebes \\ CWI, P.O. Box 94079, 1090 GB Amsterdam, The Netherlands \\ $\{c t$, arno $\} @ c w i . n l$
}

\begin{abstract}
This article presents an approach to schema integration that combines structural aspects and behavioural aspects. The novelty of the approach is that it uses behavioural information to guide both schema restructuring and schema merging. Schema restructuring is based on schema transformations and schema merging is based on join operators.
\end{abstract}

\section{Introduction}

Schema integration is an important and non-trivial task in database design. It occurs when a number of different user views, developed for a new database system, or a number of existing database schemas must be integrated into a global, unified schema. As schema integration is a difficult task, methods to support the designer with this task are essential. In [6], a framework for comparing integration methods is given. The framework identifies four steps. In the first step, the preintegration step, an integration strategy is chosen and additional information on the schemas is gathered. Subsequently, the schemas are analysed and compared to find similarities/conflicts among the schemas. In the conforming step, the conflicts found in the comparison step have to be resolved. Finally, in the last step, the schemas are merged by superimposition and the resulting schema is analysed and restructured if necessary.

For our purpose, the main characteristic of an integration method is: which similarities/conflicts are detected and how are conflicts resolved? A number of integration methods use assertions among different component schemas to compare attributes and entity types. In [17], interschema assertions, names, and types are used to compare object types. In [15], schemas are merged using schema operators and assertions among entity types and attributes in different schemas. And in [13], attribute assertions (e.g., key/non-key and lower/upper bounds) are used to compare attributes and entity types. However, the assertions must be supplied by the designer and the resolution of conflicts depends heavily on the common sense of the designer. Other methods use schema transformations to resolve structural conflicts. In [9], structural transformations are defined to integrate compatible structures. In [16], a number of

*This research is partly funded by the Dutch Organisation for Scientific Research through NFIgrant NF74. 
schema transformations (e.g., join and meet) are proposed to restructure schemas. And in [5], transformations between attributes, entities and relationships are used to resolve type conflicts. However, only the last one gives a heuristic (viz., concept likeness/unlikeness) for applying the transformations. Finally, a number of recent methods use more specific information on semantical properties of attributes and entity types to detect similarities and conflicts. In $[18,23]$, attribute assertions are used to define relationships between an attribute on one hand and a semantic point or a set of concepts on the other hand. Again, the assertions must be supplied by the designer. In [19], a database metadictionary is used to define a semantic domain for each attribute. And in [10], a terminological knowledge base containing information on negative and positive associations between terms and information on specialisation of terms is used to compare entity types.

This article presents a new approach to schema integration, based on schema transformations and the approach taken in [20,21], where classes are compared by structure and by behaviour. The approach consist of two steps. First, component schemas are restructured using schema transformations, and syntactical properties of methods are used to guide the restructuring process. Subsequently, the component schemas are merged using join operators, and semantical properties of methods are used to guide the merging process. More details on the approach are given in [22]. There is, as far as the authors know, no other approach that uses methods to compare attributes. For sake of completeness, it should be mentioned that there is an approach to schema evolution that analyses methods ([8]), not to compare attributes, but to solve non-legitimate overriding of methods.

The outline of this article is as follows. In the next section, we give a brief overview and formalisation of our data model. In Section 3, we define a number of well-known type transformations and extend them to be applicable to recursive types as well. Furthermore, we show how these type transformations induce schema transformations. In Section 4, we show how methods can be used to guide schema restructuring and give a heuristic algorithm to restructure and merge schemas. In the last section, we summarise and give some directions for further research.

\section{Database schemas}

In this section, we introduce a subset of the database schemas found in object-oriented database languages such as Galileo [2], Goblin [12], $\mathrm{O}_{2}$ [14], and TM/FM [4].

Informally, an object-oriented database schema is a class hierarchy, i.e., a set of classes related by a subclass relation. A class has a name, a set of superclasses, a set of attributes, a set of constraints, and a set of update and query methods.

Definition 1 (Class hierarchies). First, five disjoint sets are postulated: a set $C N$ of class names, a set $A N$ of attribute names, a set $M N$ of method names, a set $L$ of labels, and a set Cons of basic constants (i.e., 'integer', 'rational', and 'string' constants). The sets are generated by the nonterminals CN, AN, MN, L, and Cons, respectively. Class hierarchies are the sentences of the following BNF-grammar, where the plus sign $\left(^{+}\right)$denotes a finite, nonempty repetition, square brackets ([ ]) denote an option, and the vertical bar ( $\mid)$ denotes a choice: 


\begin{tabular}{|c|c|c|}
\hline Hierarchy & $::=$ & Class $^{+}$ \\
\hline Class & $::=$ & $\begin{array}{l}\text { 'Class' CN [ 'Isa' CN }{ }^{+} \text {] } \\
\left.\text { [ 'Attributes' Att }{ }^{+}\right] \\
\text {[ 'Constraints' Key } \\
\left.\text { ['Methods' Meth }{ }^{+}\right] \\
\text {'Endclass' }\end{array}$ \\
\hline Att & $::=$ & AN ':’ Type \\
\hline Type & $::=$ & BasicType | SetType | RecordType | CN \\
\hline BasicType & $::=$ & 'integer' | 'rational' | 'string' \\
\hline SetType & $::=$ & ' $\{$ ' Type ' $\}$ ' \\
\hline RecordType & $::=$ & '<' FieldList '>' \\
\hline FieldList & $::=$ & Field | Field ',' FieldList \\
\hline Field & $::=$ & L':’ Type \\
\hline Key & $::=$ & key KeyAtt ${ }^{+}$ \\
\hline KeyAtt & $::=$ & AN | KeyAtt '? L \\
\hline Meth & $::=$ & $\begin{array}{l}\text { MN '(' [ ParList ] ') =' AsnList | } \\
\text { MN '(' [ ParList ] } \rightarrow \text { ' Result ') =' AsnList }\end{array}$ \\
\hline ParList & $::=$ & Par | Par ',' ParList \\
\hline Par & $::=$ & L ':’ BasicType \\
\hline Result & $::=$ & L ':' Type \\
\hline AsnList & $::=$ & Assign | Assign ';' AsnList \\
\hline Assign & $::=$ & Dest ':=' Source | 'insert(' Source ',' Dest ')' \\
\hline Dest & $::=$ & L | AN | L '’ Dest | AN ‘' Dest \\
\hline Source & $::=$ & $\begin{array}{l}\text { 'self' | Term | Term '+' Source | Term '--' Source | } \\
\text { Term 'X' Source | Term ' } \div \text { ' Source | 'new (' CN ')' | } \\
\text { Dest '?' MN '(' ActParList ')' }\end{array}$ \\
\hline Term & $::=$ & Dest | Cons \\
\hline ActParList & $::=$ & Term | Term ',' ActParList \\
\hline
\end{tabular}

A class hierarchy is well-defined if it satisfies four conditions. The first condition is that the Isa relation is acyclic, and classes have a unique name and only refer to classes in the class hierarchy. The second is that attributes have a unique name within their class and are well-typed. The third is that keys must be well-defined. The fourth is that methods have a unique name within their class and are well-typed.

\subsection{Underlying types}

Informally, the set of all attributes of a class consists of both the new and inherited attributes.

Definition 2 (Attributes). Let $H$ be a class hierarchy satisfying the first condition for well-defined class hierarchies. We abbreviate every class in $H$ to a 5-tuple (c, $S, A, K, M$ ), where $c$ is the name of the class, $S$ is the set of (names of) superclasses, $A$ is the set of new attributes, $K$ is the set of new keys, and $M$ is the set of new methods. Now let $C=(c, S, A, K, M)$ be an abbreviated class in $H$. The name of $C$ is denoted by name $(C)$ and the set of all attributes of $C$, denoted by atts $(C)$, is defined as: 


$$
\begin{aligned}
\operatorname{atts}(C)=A \cup\{a: T \mid & \text { inherits }(a) \wedge \\
& \left.T=\Pi\left\{T^{\prime} \mid \text { inherits }\left(a, T^{\prime}\right)\right\} \wedge \forall a^{\prime}: T^{\prime} \in A\left[a \neq a^{\prime}\right]\right\}
\end{aligned}
$$

where

$$
\begin{aligned}
& \text { inherits }\left(a, T^{\prime}\right)=\exists C^{\prime} \in H\left[\text { name }\left(C^{\prime}\right) \in S \wedge a: T^{\prime} \in \operatorname{atts}\left(C^{\prime}\right)\right] \\
& \text { inherits }(a)=\exists C^{\prime} \in H \exists a^{\prime}: T^{\prime} \in \operatorname{atts}\left(C^{\prime}\right)\left[\operatorname{name}\left(C^{\prime}\right) \in S \wedge a=a^{\prime}\right],
\end{aligned}
$$

and $\Pi\left\{T_{1}, \cdots, T_{n}\right\}$ is the meet of a set of types [7]. Since we require that the Isa relation is acyclic, atts is well-defined.

Every class in a class hierarchy has an underlying type, which describes the structure of the class, i.e., the structure of the objects in its extensions (cf. TM/FM [4]). The underlying type of a class is an aggregation of its attributes, where recursive types [3] are used to cope with attributes that refer to classes.

Definition 3 (Underlying types). First, postulate a new type 'oid', whose extension is an enumerable set of object identifiers. Let $H$ be a class hierarchy satisfying the first condition, $C$ be a class in $H$, and $c$ be the name of $C$. The underlying type of class $C$, denoted by type $(C)$, is defined as:

$$
\text { type }(C)=\tau(c, \emptyset)
$$

where

$$
\begin{aligned}
& \tau(d, \eta)=\mu t_{d} .<\mathrm{id}: \text { oid, } a_{1}: \tau\left(T_{1}, \eta \cup\{d\}\right), \cdots, a_{k}: \tau\left(T_{k}, \eta \cup\{d\}\right)> \\
& \quad \text { if } d \notin \eta \text { and } \exists D \in H\left[n a m e(D)=d \wedge \text { atts }(D)=\left\{a_{1}: T_{1}, \cdots, a_{k}: T_{k}\right\}\right], \\
& \tau(d, \eta)=t_{d} \text { if } d \in \eta \\
& \tau(B, \eta)=B \text { if } B \in \text { integer, rational, string }\}, \\
& \tau(\{U\}, \eta)=\{\tau(U, \eta)\}, \\
& \tau\left(<l_{1}: U_{1}, \cdots, l_{n}: U_{n}>, \eta\right)=<l_{1}: \tau\left(U_{1}, \eta\right), \cdots, l_{n}: \tau\left(U_{n}, \eta\right)>.
\end{aligned}
$$

The set $\eta$ contains the names of the classes for which a (recursive) type is being constructed as part of the construction of the underlying type of class $C$. If $\eta$ contains $d$, then $\tau(d, \eta)=t_{d}$ indicates a repetition of the recursive type.

Note that the underlying type of a class depends on the hierarchy.

\section{Schema transformations}

In this section, we give an overview of type transformations and show how type transformations induce schema transformations.

The basic type transformations we have chosen (viz., renaming, aggregation, and objectification) are variants of type transformations in [1]).

Definition 4 (Basic type transformations). Let $L^{\prime}$ be the union of $L$ and $A N$ and Types be the set of types introduced in Definition 2. Renaming is defined as a function of type $L^{\prime} \rightarrow L^{\prime} \rightarrow$ Types $\rightarrow$ Types: 


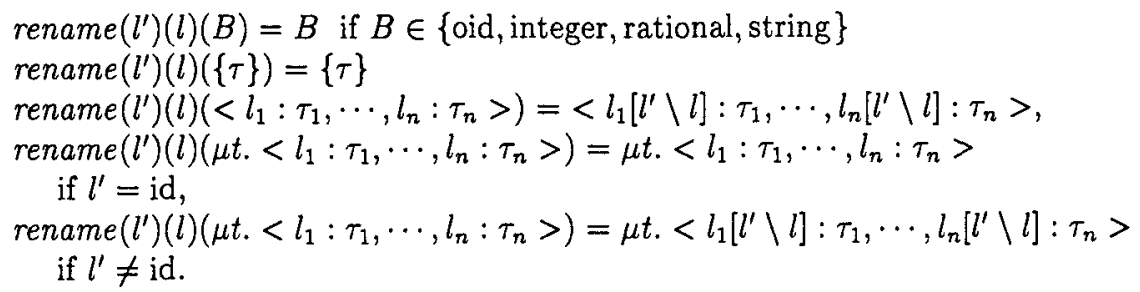

Note that we do not allow renaming of id-fields.

Aggregation is defined as a function of type $\wp\left(L^{\prime}\right) \rightarrow L^{\prime} \rightarrow$ Types $\rightarrow$ Types:

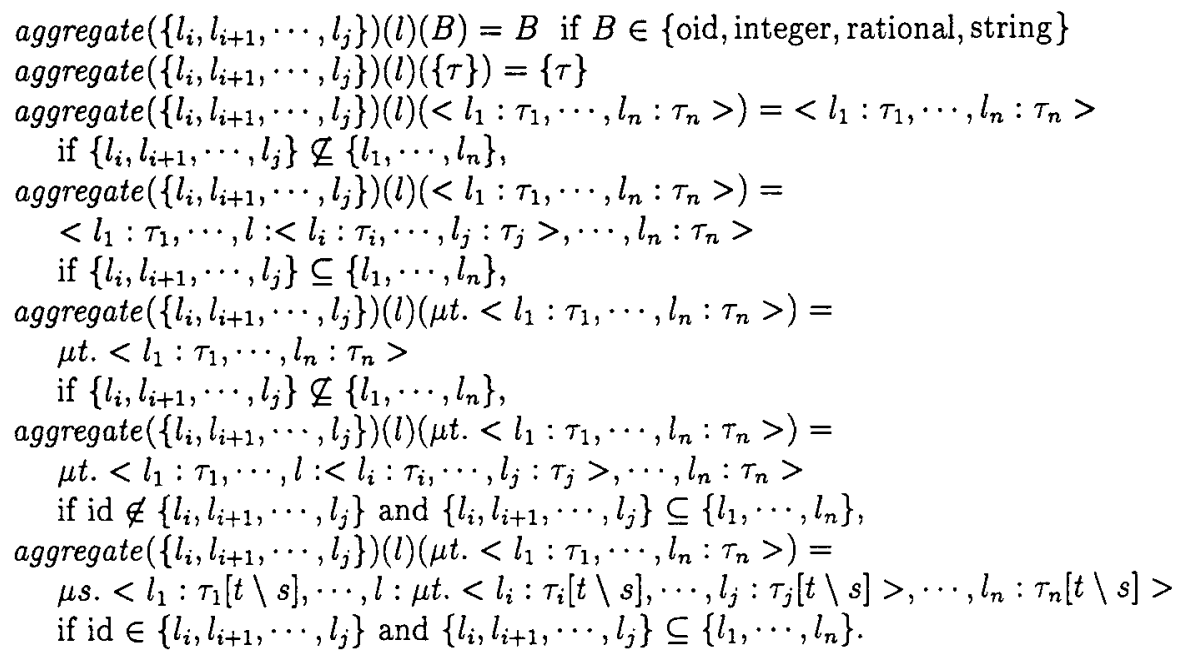

Objectification is defined as a function of type Types $\rightarrow$ Types:

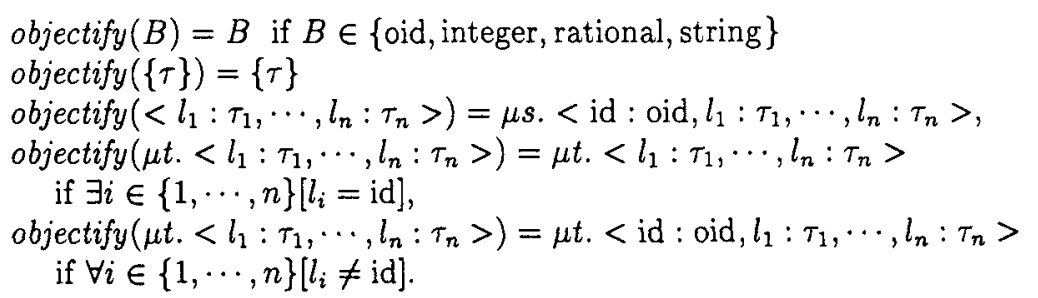

Complex type transformations are obtained by combining basic type transformations.

Example 1. Type $\sigma=\left\langle l_{1}: \mu s\right.$. <id : oid, $\left.l: \tau_{1}, l_{2}: \tau_{2}\right\rangle, l_{3}: \tau_{3}>$ can be obtained from type $\sigma_{1}=\left\langle l_{1}: \tau_{1}, l_{2}: \tau_{2}, l_{3}: \tau_{3}\right\rangle$ as follows:

$$
\begin{aligned}
& \sigma_{2}=\text { rename }\left(l_{1}\right)(l)\left(\sigma_{1}\right)=\left\langle l: \tau_{1}, l_{2}: \tau_{2}, l_{3}: \tau_{3}\right\rangle \\
& \sigma_{3}=\text { aggregate }\left(\left\{l, l_{2}\right\}\right)\left(l_{1}\right)\left(\sigma_{2}\right)=\left\langle l_{1}:<l: \tau_{1}, l_{2}: \tau_{2}\right\rangle, l_{3}: \tau_{3}> \\
& \left.\sigma_{4}=\left\langle l_{1}: \text { objectify }\left(<l: \tau_{1}, l_{2}: \tau_{2}\right\rangle\right), l_{3}: \tau_{3}\right\rangle=\sigma .
\end{aligned}
$$


Both the transformation for lexical attributes and the transformation for unstable subtypes from [11] can be obtained by composing one aggregate and one objectify operation.

Example 2. The following class hierarchy introduces a class Person, a class Employee, which inherits from class Person, and a class Company:

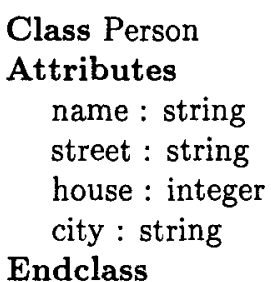

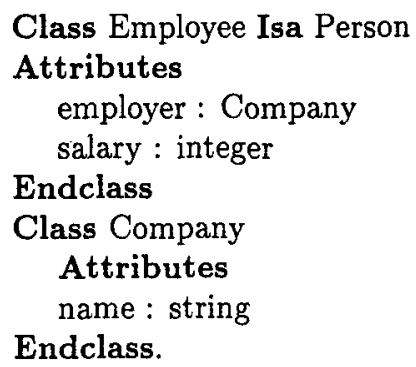

The underlying type of class Person is:

$\mu t_{P}$. <id:oid, name:string, street:string, house:integer, city:string>.

The underlying type of class Person can be transformed into (by applying aggregate ( $\{$ street,house,city $\})$ (address)):

$\mu t_{P}$. <id:oid, name:string, address:<street:string, house:integer, city:string>>, which can be transformed into (by applying objectify to the type of address):

$\mu t_{P} .<$ id:oid, name:string, address: $\mu t_{X}$. <id: oid, street:string, house:integer, city:string $>>$.

The composite transformation is a variant of the transformation for lexical attributes from [11]. We can redefine class Person as a class (named Personl) that refers to a new class (named X).

\section{Class Person 1 Attributes \\ name : string \\ address : $\mathrm{X}$ \\ Endclass}

\author{
Class X \\ Attributes \\ street : string \\ house : integer \\ city : string \\ Endclass.
}

The underlying type of class Employee is:

$\mu t_{E}$. <id:oid, name:string, street:string, house:integer, city:string, employer: $\tau_{C}$, salary:integer $>$,

where $\tau_{C}$ is the underlying type of class Company. The underlying type of class Employee can be transformed into (by applying aggregate (\{id,name,street,house,city $\}$ ) (employee)):

$\mu t_{Y}$. <employee: $\mu t_{E}$. <id:oid, name:string, street:string, house:integer, city:string $>$, employer: $\tau_{C}$, salary:integer $>$,

which can be transformed into (by applying objectify):

$\mu t_{Y}$. <id:oid, employee: $\mu t_{E}$. <id:oid, name:string, street:string, house:integer, city:string $>$, employer: $\tau_{C}$, salary:integer $>$.

The composite transformation is a variant of the transformation for unstable subtypes from [11]. We can redefine class Employee as a 'relation' (named Y) that refers to a new class (named Employee1): 


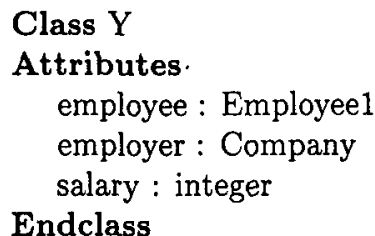

\author{
Class Employee1 \\ Attributes \\ name : string \\ street : string \\ house : integer \\ city : string \\ Endclass.
}

Note that, in the original situation, an employee (an object in class Employee), does have a unique employer, whereas, in the resulting situation, an employee (an object in class Employee1) does not. Therefore, we define a key for class Y:

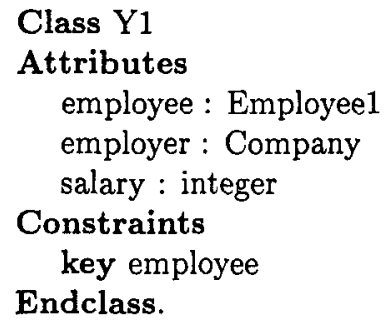

\section{Application of schema transformations}

In the previous section, we defined type transformations and showed how they induce schema transformations. In this section, we show how behaviour of methods can be used to choose among a set of schema transformations.

A class can be transformed in several ways, using different factors and different transformations.

Example 3. Let class Employee be the following class:

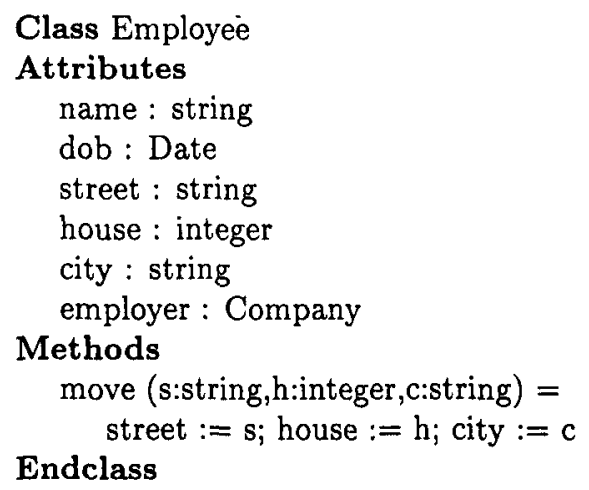

and class Address be a factor of Employee: 


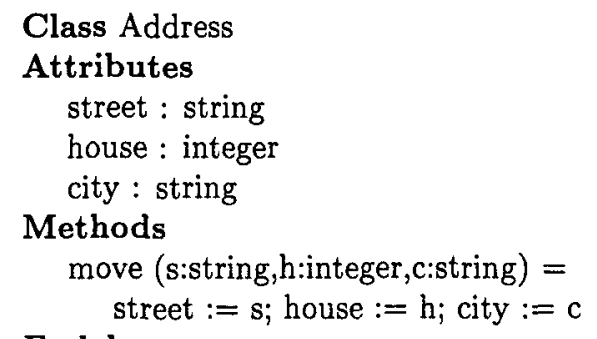

\section{Endclass.}

One option to transform class Employee is to redefine Employee as a subclass of Address (factorisation by specialisation):

\section{Class Employee1 Isa Address}

\section{Attributes}
name : string
dob : Date
employer: Company

\section{Endclass.}

Another option is to redefine Employee as a class referring to Address (factorisation by delegation):

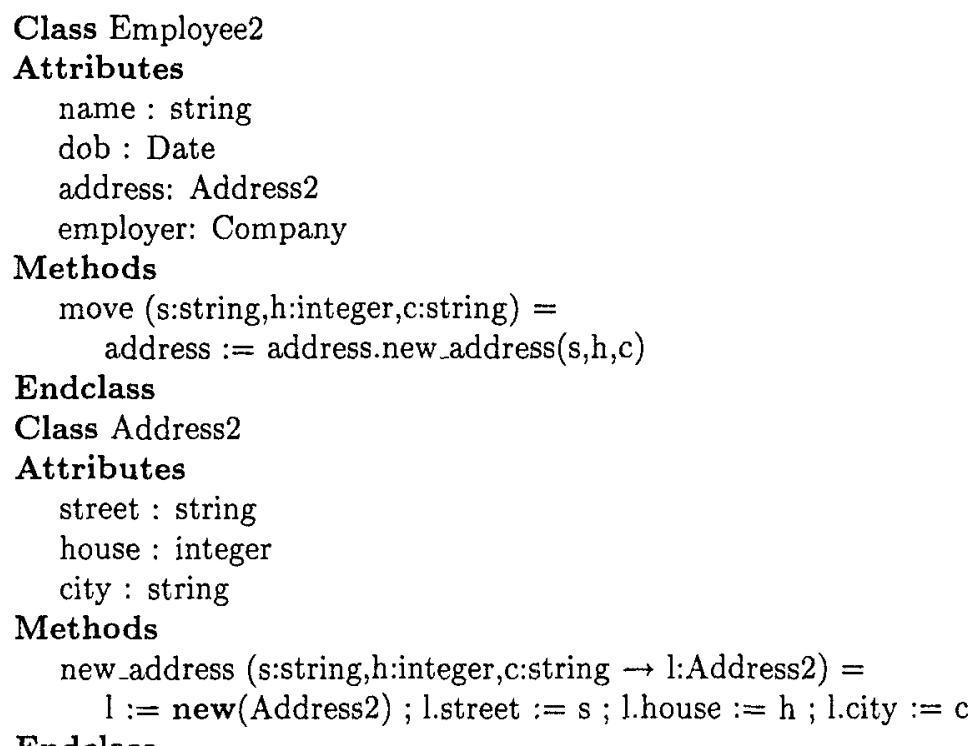

\section{Endclass.}

Note that, as an employee is not an address in the real world, it is unlikely that the first option is the right choice. The second option, where employee refers to an address (as one of its attributes) is a more reasonable choice. Now, let class Person be a factor of class Employee2:

Class Person

Attributes 


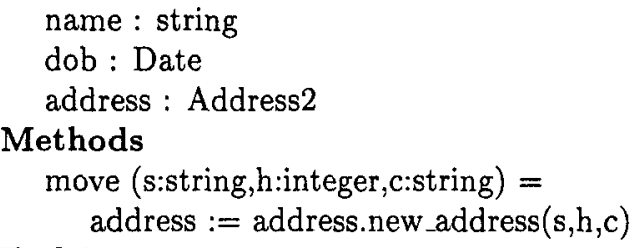

\title{
Endclass.
}

One option to transform class Employee2 is to redefine Employee2 as a subclass of Person (factorisation by specialisation):

Class Employee3 Isa Person

\author{
Attributes \\ employer : Company \\ Endclass.
}

Another option is to redefine Employee2 as a class referring to Person (factorisation by delegation):

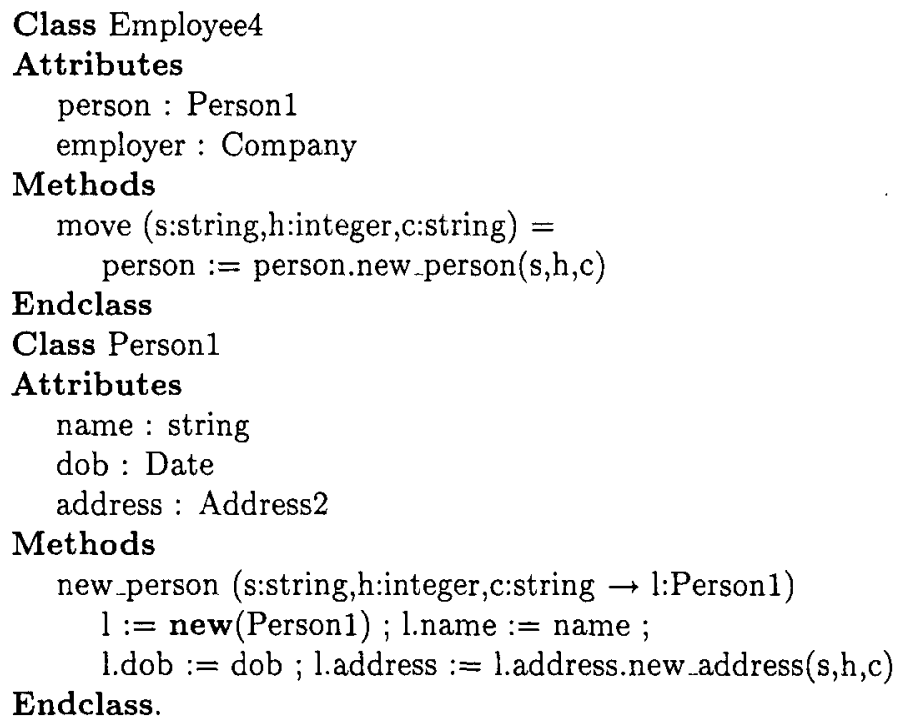

Since the objects in class Employee2 become the objects in class Employee4, we redefine method 'move' to be applicable to objects in class Employee4. Yet another option is to redefine class Employee2 as a relation involving class Person:

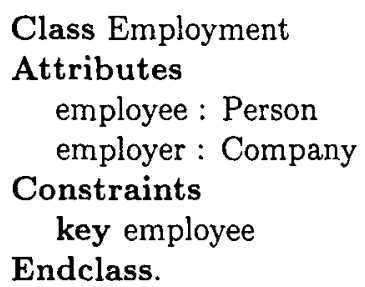


Since the objects in class Employee2 become the objects in class Person, we do not redefine method 'move', because it is already applicable to objects in class Person.

Note that, as an employee is a person in the real world, it is likely that options one and three are more reasonable than option two, where an employer refers to a person (as one of its attributes).

As we have seen, a class can be transformed in several ways, using different factors and different transformations, e.g., factorisation by specialisation, factorisation by delegation, or redefinition as a relation. But how do we choose factors and how do we choose between specialisation, delegation and redefinition as a relation? For that purpose, we introduce evidence ratios for relatedness. Weak relatedness for a set of attributes says whether the attributes are mutually related (according to the methods). Strong relatedness for a set of attributes says whether the attributes are mutually related, but not to attributes outside the set (according to the methods). Isolation for a set of attributes says whether the attributes are not related to attributes outside the set (according to the methods).

Definition 5 (Relatedness ratios). Let $H$ be a well-defined class hierarchy, $C$ be a class in $H, c$ be the name of $C$, and $M$ be the set of all methods of $C$. Furthermore, for meth $\in M$, let atts (meth) consist of the names of attributes of $C$ that occur in meth. Weak relatedness of a set of attributes $A \subseteq\{a \mid a: T \in$ atts $(C)\}$ is defined as:

$$
\text { weakrel }(c, A)=\frac{\mid\{\text { meth } \in M \mid \text { atts }(\text { meth }) \supseteq A\} \mid}{\mid\{\text { meth } \in M \mid \text { atts }(\text { meth }) \cap A \neq \emptyset\} \mid} .
$$

Strong relatedness of a set of attributes $A$ is defined as:

$$
\text { strongrel }(c, A)=\frac{\mid\{\text { meth } \in M \mid \text { atts }(\text { meth })=A\} \mid}{\mid\{\text { meth } \in M \mid \text { atts }(\text { meth }) \cap A \neq \emptyset\} \mid} .
$$

Isolation of a set of attributes $A \subseteq\{a \mid a: T \in \operatorname{atts}(C)\}$ is defined as:

$$
\text { isolation }(c, A)=\frac{\mid\{\text { meth } \in M \mid \emptyset \neq \text { atts }(\text { meth }) \subseteq A\} \mid}{\mid\{\text { meth } \in M \mid \text { atts }(\text { meth }) \cap A \neq \emptyset\} \mid} .
$$

If $\{$ meth $\in M \mid$ atts $($ meth $) \cap A \neq \emptyset\}$ is empty, then weakrel $(c, A)$ and $\operatorname{strongrel}(c, A)$ are defined to be 0 , and isolation $(c, A)$ is defined to be 1 .

For a set of attributes with strong relatedness ratio 1 and any method, either all attributes occur in the method and all attributes that occur in the method are in the set, or no attribute in the set occurs in the method. In that case, the attributes are strongly related. For a set of attributes with weak relatedness ratio 0 , there is no method in which all attributes occur and, hence, the attributes are not (mutually) related. And for a set of attributes with isolation ratio 1 and any method, either all attributes that occur in the method are attributes in the set or no attribute that occurs in the method is an attribute in the set. In that case, the attributes are only related within the set.

Weak and strong relatedness can help to choose a factor. If the strong relatedness ratio of a set of attributes is high, then it is reasonable to believe that they belong together and, hence, to factorise. On the other hand, if the weak relatedness ratio is low, then it reasonable to believe that they do not belong together and, hence, not to factorise. 
Example 4. Consider class Employee of Example 3. The weak and strong relatedness ratios for \{street, house, city\} and \{name, dob\} are given by:

strongrel (Employee, $\{$ street, house, city $\})=1$

weakrel (Employee, $\{$ street, house, city $\})=1$

strongrel (Employee, $\{$ name, dob $\})=0$

weakrel (Employee, $\{$ name, dob $\})=0$.

As we can see, street, house, and city are strongly related, whereas name and dob are not related.

Now, consider class Employee2 of Example 3. The weak and strong relatedness ratios for \{name, dob, address\} and \{name, dob, employer\} are given by:

strongrel(Employee2, \{name, dob, address $\})=0$

weakrel (Employee2, \{name, dob, address $\})=0$

strongrel (Employee2, \{name, dob, employer $\})=0$

weakrel (Employee2, $\{$ name, dob, employer $\})=0$.

As we can see, in both cases the attributes are not related.

Isolation can help to choose between specialisation and redefinition as a relation. If the isolation ratio is less than one, then specialisation is possible, but redefinition as a relation is not, since, in that case, we have to add a method to the relation that updates another relation or class.

Example 5. Consider class Employee2 of Example 3. The isolation evidence ratio for name, dob, address is given by:

isolation (Employee2, \{name, dob, address $\})=1$.

Redefinition as a relation results in a relation (Employment) that represents a simple association between a person and a company. Now, if we add a method to class Employee2 that updates attribute address and attribute employer, then we will have to add a method to Employment that creates a new person and updates attribute employee and attribute employer. Since this method is not a simple insert or update operation on Employment, Employment is no longer a relation.

So, how do we choose factors and transformations? Factors are chosen by comparing weak evidence ratios. If the weak evidence ratio of a set of attributes is greater than some threshold, there is reason to assume that the attributes can be used as a factor. If not, there is no reason. Transformations are chosen by comparing strong evidence ratios and isolation ratios. In case the strong evidence ratio is greater than some threshold, delegation is a reasonable option, because the attributes are strongly related within the set and weakly related with other attributes. In case the isolation ratio is less than one, then specialisation is possible, but redefinition as a relation is not. Otherwise, specialisation or redefinition as a relation are both possible. It should be mentioned that, in the context of schema integration, schema transformations must be applied carefully and only if necessary. In particular, this is true for factorisation by specialisation, since a lot of new classes will be generated by this type of transformation.

The considerations for choosing factors and transformations can be used in a heuristic algorithm to support schema integration. First, the attributes of every class are partitioned in such a way that the isolation ratio of every element in the partition is one, and every class is factorised by delegation if desirable. Subsequently, for every 
pair of promising classes, a set of possible superclasses is computed, and both classes are factorised by specialisation or redefined as a relation if desirable.

Algorithm 1. The following algorithm is a heuristic for integrating two database schemas (resp., DBS1 and DBS2), given thresholds for strong relatedness and weak relatedness (resp., TSR and TWR):

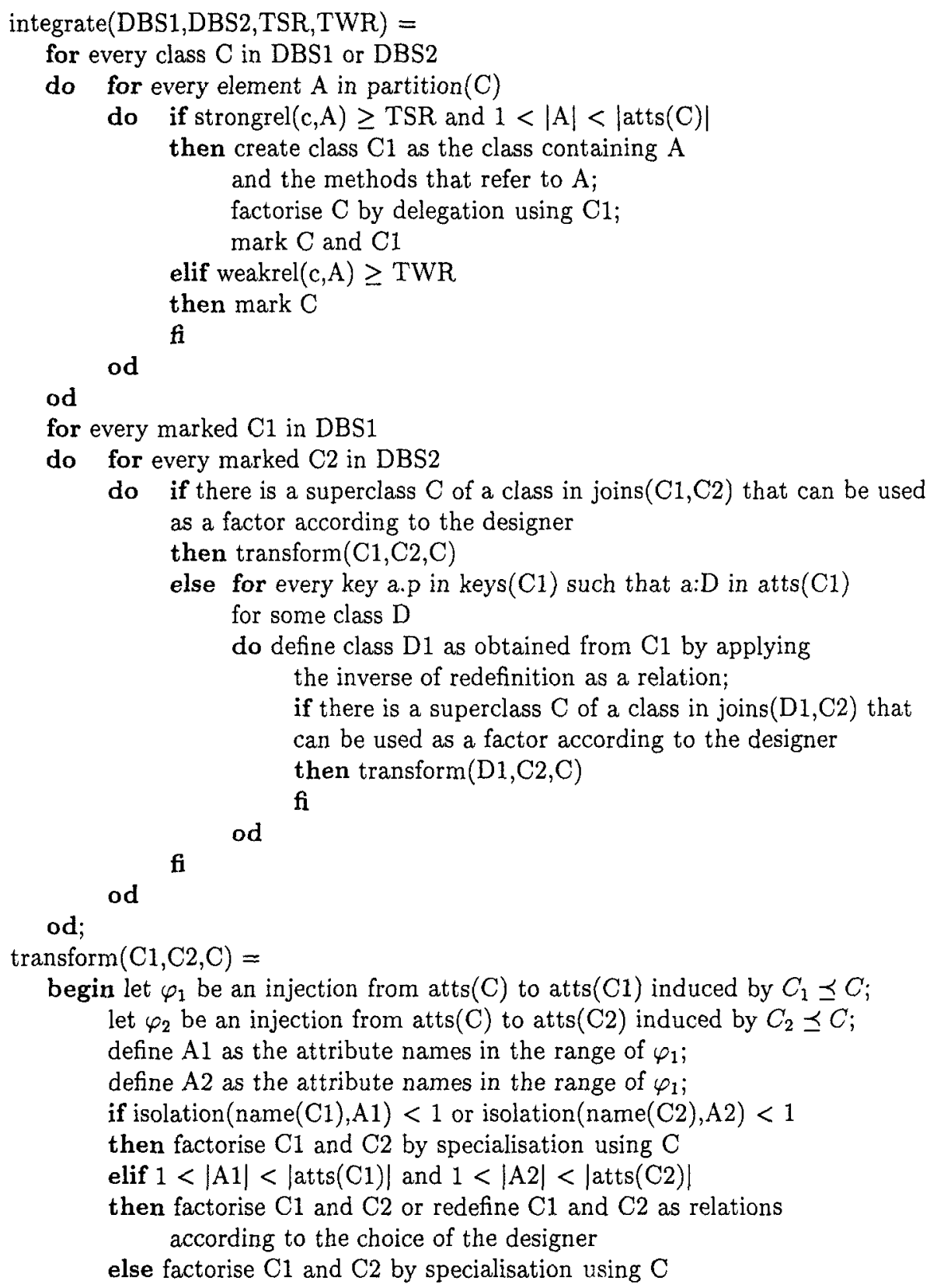

begin let $\varphi_{1}$ be an injection from $\operatorname{atts}(C)$ to atts(C1) induced by $C_{1} \preceq C$;

let $\varphi_{2}$ be an injection from $\operatorname{atts}(\mathrm{C})$ to $\operatorname{atts}(\mathrm{C} 2)$ induced by $C_{2} \preceq C$;

define $A 1$ as the attribute names in the range of $\varphi_{1}$;

define $A 2$ as the attribute names in the range of $\varphi_{1}$;

if isolation(name $(\mathrm{C} 1), \mathrm{A} 1)<1$ or isolation(name $(\mathrm{C} 2), \mathrm{A} 2)<1$

then factorise $\mathrm{C} 1$ and $\mathrm{C} 2$ by specialisation using $\mathrm{C}$

elif $1<|\mathrm{A} 1|<|\operatorname{atts}(\mathrm{C} 1)|$ and $1<|\mathrm{A} 2|<|\operatorname{atts}(\mathrm{C} 2)|$

then factorise $\mathrm{C} 1$ and $\mathrm{C} 2$ or redefine $\mathrm{C} 1$ and $\mathrm{C} 2$ as relations according to the choice of the designer

else factorise $\mathrm{C} 1$ and $\mathrm{C} 2$ by specialisation using $\mathrm{C}$ 
end;

where partition(C) is constructed as follows:

$\operatorname{graph}(\mathrm{C})$ has a node for every attribute name in $\operatorname{atts}(\mathrm{C})$

graph(C) has an edge between two nodes if there is a method in the set of all methods of $C$ in which both attribute names occur

partition(C) consists of sets of attribute names, one set for every connected subgraph of graph(C): two attribute names are in the same set if their nodes are connected two attribute names are in different sets if their nodes are not connected,

and joins(D1,D2) (i.e., the set of common superclasses of D1 and D2) and $\preceq$ (i.e., the subclass relation) are as defined in [21].

Note that the algorithm interacts with the designer. It should be mentioned again that the algorithm is a heuristic and should therefore be used in close interaction with the designer. The heuristic can be improved by combining the different thresholds and refining the different actions. This is the subject of future research.

\section{Conclusion}

In this article, we presented a new approach to schema integration based on transformations and behaviour. First, we formalised schemas using underlying types and underlying constraints. Next, we presented a number of type transformations on underlying types and used them to transform schemas. Finally, we gave a heuristic algorithm for integrating schemas. The algorithm uses schema transformations to restructure schemas and join operators to merge them and behavioural information to guide restructuring and merging. Advantages of this approach are: 1) structural aspects are integrated in a guided fashion and 2) both structural and behavioural aspects are integrated.

Further research includes 1) extension of the data model, 2) extension of the schema transformations, and 3) extension and refinement of the heuristic algorithm.

\section{Bibliography}

[1] S. Abiteboul and R. Hull. Restructuring hierarchical database objects. Theoretical Computer Science, 62:3-38, 1988.

[2] A. Albano, L. Cardelli, and R. Orsini. Galileo: A strongly typed, interactive conceptual language. ACM Trans. on Database Systems, 10(2):230-260, 1985.

[3] R. Amadio and L. Cardelli. Subtyping recursive types. In Proc. Int. Symp. on Principles of Programming Languages, pages 104-118, 1991.

[4] P. Apers, H. Balsters, R. de By, and C. de Vreeze. Inheritance in an object-oriented data model. Memoranda Informatica 90-77, University of Twente, Enschede, The Netherlands, 1990.

[5] C. Batini and M. Lenzerini. A methodology for data schema integration in the ER model. IEEE Transactions on Software Engineering, pages 650-664, November 1984. 
[6] C. Batini, M. Lenzerini, and S. Navathe. A comparative analysis of methodologies for database schema integration. ACM Computing Surveys, 18(4):323-364, 1986.

[7] L. Cardelli. A semantics of multiple inheritance. In Proc. Int. Symp. on Semantics of Datatypes, LNCS 173, pages 51-67. Springer-Verlag, Berlin, 1984.

[8] E. Casais. An incremental class reorganization approach. In European Conf. on ObjectOriented Programming, pages 114-132, 1992.

[9] R. Elmasri and G. Wiederhold. Data model integration using the structural model. In Proc. Int. Conf. on Management of Data, pages 191-202, 1979.

[10] P. Fankhauser, M. Kracker, and E. Neuhold. Semantic vs. structural resemblance of classes. ACM SIGMOD Record, 20(4):59-63, 1991.

[11] P. Johannesson. Schema transformations as an aid in view integration. In Proc. Int. Conf. on Advanced Information Systems Engineering, LNCS 685, pages 71-92. Springer-Verlag, Berlin, 1993.

[12] M. Kersten. Goblin: a DBPL designed for advanced database applications. In Proc. Int. Conf. on Databose and Expert Systems Applications, pages 345-349. Springer-Verlag, Wien, 1991.

[13] J. Larson, S. Navathe, and R. Elmasri. A theory of attribute equivalence in databases with application to schema integration. IEEE Transactions on Software Engineering, 15(4):449-463, 1989.

[14] C. Lécluse and $\mathrm{P}$. Richard. The $\mathrm{O}_{2}$ database programming language. In Proc. Int. Conf. on Very Large Databases, pages 411-422. Morgan Kaufmann, Palo Alto, CA, 1989.

[15] M. Mannino, S. Navathe, and W. Effelsberg. A rule based approach for merging generalisation hierarchies. Information Systems, 13(3):257-272, 1988.

[16] A. Motro and P. Buneman. Constructing superviews. In Proc. Int. Conf. on Management of Data, pages 56-64, 1981.

[17] S. Navathe and S.Gadgil. A methodology for view integration in logical data base design. In Proc. Int. Conf. on Very Large Databases, pages 142-155, 1982.

[18] A. Sheth and S. Gala. Attribute relationships: an impediment in automating schema integration. In Proc. Workshop on Heterogeneous Database Systems, 1989.

[19] M. Siegel and S. Madnick. A metadata approach to resolving semantic conflicts. In Proc. International Conference on Very Large Databases, pages 133-145, 1991.

[20] C. Thieme and A. Siebes. Schema integration in object-oriented databases. In Proc. Int. Conf. on Advanced Information Systems Engineering, LNCS 685, pages 54-70. Springer-Verlag, Berlin, 1993.

[21] C. Thieme and A. Siebes. Schema refinement and schema integration in object-oriented databases. In Proc. Computing Science in The Netherlands, ISBN 906196430 X, pages 343-354. Stichting Mathematisch Centrum, 1993.

[22] C. Thieme and A. Siebes. An approach to schema integration based on transformations and behaviour. Report CS-R9403, CWI, Amsterdam, The Netherlands, 1994 (available by anonymous ftp from ftp.cwi.nl).

[23] C. Yu, W. Sun, S. Dao, and D. Keirsey. Determining relationships among attributes for interoperability of multi-database systems. In Proc. Int. Workshop on Interoperability in Multidatabase Systems, pages 251-257, 1991. 